\title{
Estimation of respiration rate using an accelerometer and thermal camera in eGlasses
}

\author{
Jacek Ruminski Member, IEEE, Adam Bujnowski, Krzysztof Czuszynski, Tomasz Kocejko \\ Gdansk University of Technology \\ Gdansk, Poland \\ jacek.ruminski@pg.gda.pl
}

\begin{abstract}
Respiration rate is a very important vital sign. Different methods of respiration rate measurement or estimation have been developed. However, especially interesting are those that enable remote and unobtrusive monitoring. In this study, we investigated the use of smart glasses for the estimation of respiration rate especially useful for indoors applications. Two methods were analyzed. The first one is based on measurements of respiration-related body movements using an accelerometer. The second one uses the thermal camera to observe temperature changes in the nostril region. For both methods signals were extracted, filtered and processed using two different respiration rate estimators. Both methods were validated during experiments with the participation of volunteers using the respiration belt as a reference measurement method. Results proved that for both methods it is possible to reliable estimate the respiration rate with Root Mean Square Error lower than 2 breaths per minute, which is sufficient for medical screening.
\end{abstract}

Keywords-smart glasses; respiration rate estimation, thermal imaging

\section{INTRODUCTION}

Smart glasses are wearable devices that can extend human senses and capabilities of information processing. Additionally, the near-to-eye display could provide graphical information with much higher privacy than a smartphone or a tablet. Smart glasses can be equipped with different sensors (e.g. accelerometers, gyroscopes, cameras), communication interfaces (e.g. WiFi, Bluetooth), etc. Recently, many devices have been proposed on the consumer market, including Google Glass, Epson Moverio BT-200, Recon Jet, Lumus DK40, etc. [1]. Many ideas and demonstrations of potential roles of smart glasses in healthcare have been presented. Some include improved visualization of veins locations (Evena Medical [2]), access and visualization of data from medical records [3][4][5], presentation of vital signs on the display of smart glasses (Philips [6]), etc.

In this paper we analyze the possible role of smart glasses in estimation of respiration rate. Smart glasses were previously used to estimate some vital signs [7][8]. Typically, the pulse rate was estimated using photoplethysmography [9] or respiration rate (and pulse rate) using data collected by an accelerometer or gyroscope of the Google Glass [7]. Additionally, for the identified patient (e.g. using face recognition [10] or using graphical markers like QR-code [11][12]) the measured vital signs or other health-related data can be automatically uploaded to the healthcare information

This work has been partially supported by NCBiR, FWF, SNSF, ANR and FNR in the framework of the ERA-NET CHIST-ERA II, project eGLASSES - The interactive eyeglasses for mobile, perceptual computing and by Statutory Funds of Electronics, Telecommunications and Informatics Faculty, Gdansk University of Technology. system. The goal of this paper is also to analyze two different respiration rate estimators applied to short-time data collected using the accelerometer or the thermal camera. Respiration rate is typically monitored using masks with thermistors, analyzing ECG drifts or using clinical observations. It is especially important in quality of sleep analysis (e.g. for sleep apnea detection [13]). Some remote methods use analysis of video sequences recorded from the chest region [14] looking for respiration-related movements. Those recordings are typically performed using visible light cameras [15] and infrared cameras [16][17]. Thermal recordings were typically performed using cameras with good spatial resolution [18][19] performing respiratory rate analysis in the frequency domain.

The rest of the paper is structured as follows: Section II presents the proposed methods. Results are described in Section III. Section IV present a discussion of results and concludes the paper.

\section{MethodS}

\section{A. Measurement systems}

Respiration is important measurement of the body's most fundamental function. Smart glasses can use different sensors to measure or estimate respiration rate (for the observed person and for the wearer). Here, we assume that respiration rate can be estimated using analysis of data captured: with accelerometer/gyroscope sensors - for the user of smart glasses and with the thermal camera - for the observed person. Both measurement methods were implemented in the eGlasses prototype, developed under the eGlasses project (www.eglasses.eu). This experimental platform is dedicated to research activities, so different electronic modules can be changed; it is possible to print another cover using 3D printer, add sensors or electrodes, change the display, etc. The current prototype uses OMAP 4460 processor with 1GB RAM, $1024 \times 768$ transparent display from Elvision Company, 5MPx camera, WiFi and Bluetooth 4 wireless interfaces, different sensors (accelerometer, gyroscope, magnetometer, OMRON D6T thermal sensor, etc.), eye-tracker and extension slots. The Android 4.1 OS and Linux Ubuntu OS have been already tested. For the goals of this paper the accelerometer and the thermal camera module were used.

\section{B. Accelerometer and respiration rate}

The used single-chip MPU-6500 (Invesense) integrates the 3 -axis accelerometer, the 3-axis gyroscope, and the onboard Digital Motion Processor ${ }^{\mathrm{TM}}$ (DMP) in a small, $3 \mathrm{~mm} \times 3 \mathrm{~mm} \times$ $0.9 \mathrm{~mm}$ package. The device can operate from $1.8 \mathrm{~V}$ and 
consumes $6.1 \mathrm{~mW}$ in full operating mode $(33 \mu \mathrm{W}$ in low-power mode). The typical offset of the accelerometer is $\pm 60 \mathrm{mg}$ and $300 \mu \mathrm{g} / \sqrt{\mathrm{Hz}}$ of noise. The location of the chip on the base board (Fig. 1) enables the measurement of acceleration in $x$ direction (head top to down), $y$ direction (back of the head towards face) and $z$ direction (ear-to-ear direction).
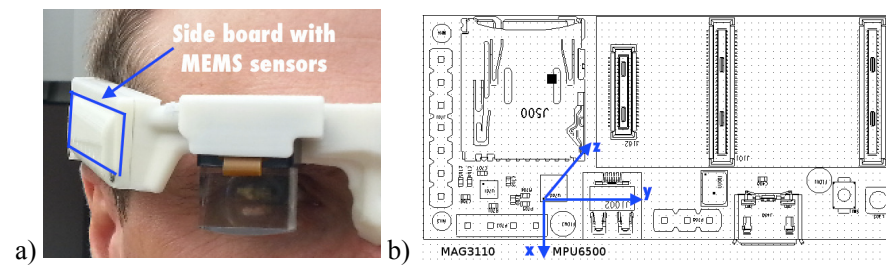

Fig. 1. a) The eGlasses prototype with the indicated location of the (side) base board with sensors. b) The layout of the (side) base board with the indicated axes of the accelerometer and gyroscope.

It is assumed that respiration activities influence body (head) movements so it should be possible to estimate the respiration rate. Typically, the acceleration module is calculated as:

$$
|a|=\sqrt{a_{x}^{2}+a_{y}^{2}+a_{z}^{2}}
$$

where: $a_{x}, a_{y}$, and $a_{z}$ are the measured acceleration values for particular directions.

In this work, measured signals ( $|a|$ or directional $a$ signals) are resampled (to $f_{s}=11 \mathrm{~Hz}$ ) and normalized (mean removal). Next, filtration is used to remove noise and not-respiratory related signal components. The moving average is used (window size $f_{s} / 2$ ) and the band-pass Butterworth filter ( $4^{\text {th }}$ order) to pass frequencies between $6 \mathrm{bpm}$ and $40 \mathrm{bpm}$. Finally, two respiratory rate estimators are applied (described later).

\section{Thermal camera and respiration rate}

The respiration rate was also analyzed using sequences of thermal images recorded for nostril regions. The FLIR Lepton thermal camera module, located in the designed front board, was used for thermal recordings (Fig. 2). It is characterized by high dynamic range (14bits), small size $\left(<1 \mathrm{~cm}^{2}\right)$ and relatively small spatial resolution $(80 \times 60)$.

The multistep procedure was used for the estimation of respiration rate. First, a sequence of thermal frames was captured during the short time period (30s windows were used in the experiments). Next, in this preliminary study, the nostrils region (a rectangle with width $=$ nose width) was manually selected directly around/below the nose. Then, the nostril ROI was used to calculate the average pixel value inside that ROI. The operation was repeated for each frame producing a 1-D signal (time series) of infrared (thermal) radiation changes. Next the same filtration was used as for signals obtained for the accelerometer (i.e. moving average and Butterworth band-pass filter).

\section{Respiration rate estimators}

Two respiration rate estimators were analyzed for signals obtained for the accelerometer and for the thermal camera. The first estimator, $e R R \_s p$, often used in other studies, identifies the frequency in the frequency domain of the analyzed signal, for the dominating peak in the frequency spectrum.

a)

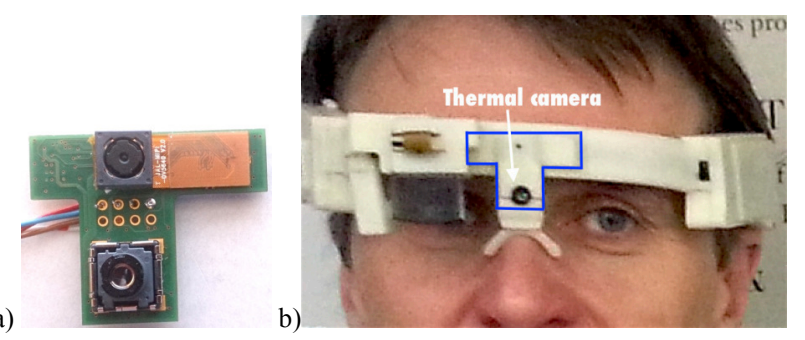

Fig. 2. a) The module with cameras: visible ligth camera (top) and Lepton infrared camera (bottom). b) The location od the module in the eGlasses.

The second estimator, $e P R \_a c$, analyzes the periodicity of peaks locations for the autocorrelation function in time domain as a function of time lags. The autocorrelation for different time lags is calculated and the period is determined calculating an average time differences between detected peaks. As a peak detector we used a method looking for a local minimum and a local maximum, for which their difference is grater than the threshold value $T$ :

$$
d=s_{f n}\left(t_{j+1}\right)-S_{f n}\left(t_{j}\right), d>T,
$$

where: $s_{f n}\left(t_{j+l}\right)$ - filtered signal value of the local minimum at $j$, $s_{f n}\left(t_{j}\right)$ - filtered signal value of the local maximum at $j+l$.

The threshold value $\mathrm{T}$ was calculated in two phases. In the first phase $\left(T=T_{l}\right)$ as:

$$
T_{I}=T_{K 1} *\left(\max \left(s_{f n}(t)\right)-\min \left(s_{f n}(t)\right)\right),
$$

where $T_{K I}$ was the scaling value set to 0.33 .

Then the median of the detected peak-to-peak gradient values was calculated. The scaled $\left(T_{K l}=0.25\right)$ median value was used as the threshold value $\left(T=T_{2}\right)$ in the second pass of the algorithm to detect final peaks of each signal.

The calculated frequencies for both estimators were multiplied by 60 to obtain results in breaths per minute (bpm, e.g. $\left.e P R \_a c=f_{a c} * 60\right)$.

\section{E. Experiments and validation}

The analyzed methods for the estimation of respiration rate were validated during experiments with the participation of 11 volunteers (mean age: $39.73 y \pm 11.98$ ). Subjects were asked to seat comfortable and not move except natural breathing. Then, separately, data were measured during $1 \mathrm{~min}$, by two devices. In parallel, the pressure, chest belt (Vernier RMB) was used as a reference. To synchronize signals subjects were asked to hold the breath at the beginning of data recording. Measured signals were analyzed manually to calculate reference respiration rate (RR) values. According to the RR definition (number of respiration events in time) the complete periods between successive inspirations were indicated and counted in 30s long time windows. The RR was calculated as:

$$
R R=\left(N_{R R} * 60\right) /\left(t_{l e}-t_{f s}\right),
$$

where: $N_{R R}$ - number of respiration events (inspiration to inspiration), $t_{l e}$ - time of the end of the last event, $t_{f s}$ - time of the start of the first event. 


\section{RESULTS}

\section{A. Accelerometer and respiration rate}

Table I presents results of the respiration rate estimation for measurements performed using the accelerometer of smart glasses and using the reference respiration belt.

TABLE I. RESULTS OF RESPIRATION RATE ESTIMATION FOR THE ACCELEROMETER

\begin{tabular}{|l|r|r|r|r|r|}
\hline & \multicolumn{3}{|c|}{ Chest Belt } & \multicolumn{2}{l|}{ Accelerometer } \\
\hline Subject & RR $[\mathrm{bpm}]$ & eRR_sp & eRR_ac & eRR_sp & eRR_ac \\
\hline S01 & 15.400 & 14.650 & 14.990 & 10.950 & 14.160 \\
\hline S02 & 21.400 & 21.970 & 21.650 & 20.480 & 20.160 \\
\hline S03 & 10.000 & 10.254 & 9.900 & 9.580 & 9.470 \\
\hline S04 & 15.670 & 16.110 & 15.740 & 8.020 & 11.160 \\
\hline S05 & 20.000 & 20.510 & 20.000 & 17.977 & 18.310 \\
\hline S06 & 15.000 & 13.184 & 13.470 & 13.840 & 13.760 \\
\hline S07 & 14.060 & 13.180 & 13.920 & 16.450 & 15.600 \\
\hline S08 & 13.240 & 13.180 & 13.370 & 13.450 & 13.370 \\
\hline S09 & 11.900 & 11.720 & 12.040 & 9.350 & 10.680 \\
\hline S10 & 13.640 & 13.180 & 13.890 & 13.610 & 13.030 \\
\hline S11 & 13.640 & 13.180 & 13.590 & 12.520 & 12.840 \\
\hline
\end{tabular}

The values of RMSE for particular estimators are: for the belt: $R M S E\left(e R R \_s p\right)=0.73, \quad R M S E\left(e R R \_a c\right)=0.50 ;$ for the accelerometer: $R M S E\left(e R R \_s p\right)=2 . \overline{99}, \operatorname{RMSE}\left(e R R \_a c\right)=1.73$. Signal examples are presented in Fig. 3: measured signals by the accelerometer (subject S05) and obtained results (spectrum for the filtered signal, and autocorrelation as a function of time lags with the detected peaks).
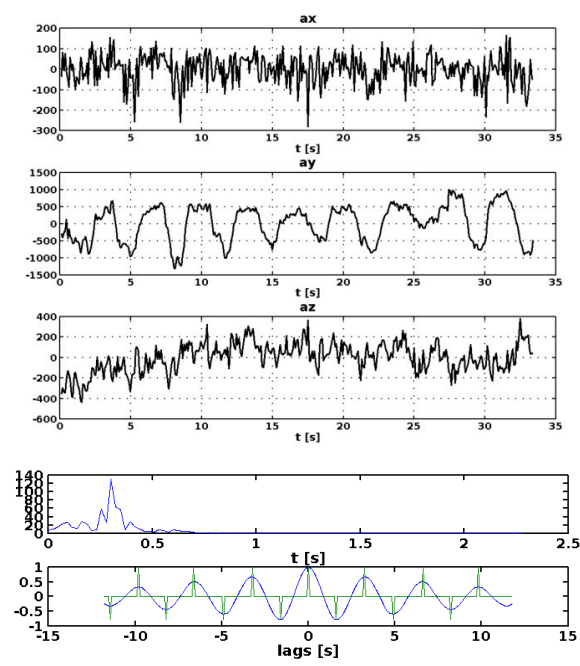

Fig. 3. Top: Signals from the accelerometer for subject S05. Bottom: Spectrum of the filterred signal and autocorrelation signal as a function of time lags with indicated detected peaks.

Table II presents results of the respiration rate estimation for measurements performed using the thermal camera of smart glasses and using the reference respiration belt.

The values of RMSE for particular estimators are: for the belt: $R M S E\left(e R R \_s p\right)=0.55, R M S E\left(e R R \_a c\right)=0.24$; for the thermal camera: $R M S E\left(e R R \_s p\right)=0.68, R M S E\left(e R R \_a c\right)=0.69$.
TABLE II. RESULTS OF RESPIRATION RATE ESTIMATION FOR THE THERMAL CAMERA

\begin{tabular}{|l|r|r|r|r|r|}
\hline & \multicolumn{2}{|l|}{ Chest Belt } & \multicolumn{2}{l|}{ Thermal camera } \\
\hline Subject & RR [bpm] & eRR_sp & eRR_ac & eRR_sp & eRR_ac \\
\hline S01 & 17.900 & 18.281 & 17.863 & 18.281 & 18.140 \\
\hline S02 & 13.000 & 12.188 & 12.893 & 13.711 & 13.448 \\
\hline S03 & 12.188 & 12.188 & 12.480 & 12.188 & 12.581 \\
\hline S04 & 18.200 & 18.281 & 18.425 & 18.281 & 18.571 \\
\hline S05 & 20.955 & 21.328 & 20.526 & 21.328 & 22.609 \\
\hline S06 & 9.600 & 10.664 & 9.936 & 10.664 & 9.936 \\
\hline S07 & 7.200 & 7.620 & 7.430 & 7.610 & 7.090 \\
\hline S08 & 19.809 & 19.805 & 19.873 & 18.281 & 18.699 \\
\hline S09 & 19.158 & 18.281 & 18.828 & 19.805 & 19.141 \\
\hline S10 & 19.711 & 19.805 & 19.623 & 19.805 & 20.526 \\
\hline S11 & 13.220 & 13.711 & 13.220 & 13.711 & 13.200 \\
\hline
\end{tabular}

In Fig. 4a some examples of thermal images captured during inspiration and expiration events are presented. In Fig. $4 \mathrm{~b}$ examples of the filtered signal of thermal radiation changes in the nostril ROI (subject S01) and obtained results (spectrum for the filtered signal, and autocorrelation as a function of time lags with the detected peaks) are presented.

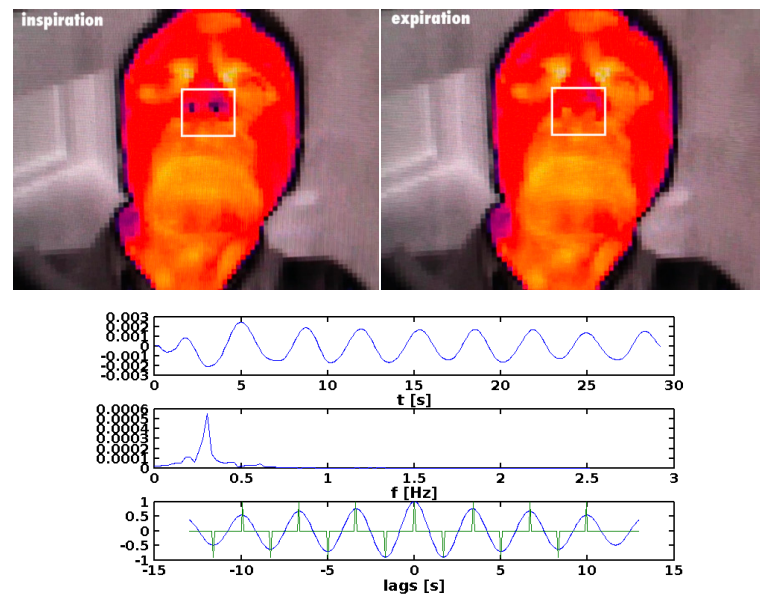

Fig. 4. Top: Thermal images for inspiration and expiration events. Bottom: Filtered signal, spectrum of the filterred signal and autocorrelation signal as a function of time lags with indicated detected peaks.

\section{DisCUSSION AND CONCLUSIONS}

In this study we proposed the use of smart glasses for the estimation of respiration rate using two sensors. The first method was based on measurements performed with the accelerometer. As it could be observed in Fig. 3a respirationrelated movements are mainly (for most participants) observed in $y$ direction (head backward/forward). This is probably related to head movement during inspiration, when the chest cage/lungs are filled with air causing natural head movement to the back. The calculated $R M S E$ value for the $e R R \_a c$ estimator shows that the respiration rate can be estimated with relatively good accuracy. This is usually good enough for medical screening purposes. For most subjects the accuracy of the respiratory rate was up to $2 \mathrm{bpm}$. However, as it could be observed for S04, in some cases the result is not acceptable. It is important to underline, that the value of respiration rate is always calculated for a particular time window. So manually 
calculated RR values for the belt-based signals are a little bit different that values automatically calculated by two estimators. This is also caused by analyzing different time windows. The manually calculate RR values considered only the whole inspiration-to-inspiration periods. Other estimators used all samples. Additionally, the estimator based on the frequency domain has limited accuracy due to the finite frequency resolution. For example, taking $N=330$ samples and $f_{s}=11 \mathrm{~Hz}$, then spectral resolution is $(11 / 330) * 60=2 \mathrm{bpm}$. For experiments with the use of the accelerometer the $e R R \_s p$ gave worse results than the $e R R \_a c$ estimator performing operations in time domain. The experiments were performed under optimistic conditions - subjects did not move. Further research is required to verify the methodology in more natural conditions (head movements).

Experiments performed for the small thermal camera module gave very good results. The respiration rate can be easily and reliably estimated using the described method. The method has of course limits related to required gradient between values of body temperature and ambient temperature. However, when used indoors the required temperature gradient is practically usually fulfilled (e.g. air-conditioning). One of the most interesting finding is that even such a small spatial resolution is not a problem for locating nostril regions and obtaining high dynamics of signals (ambient temperature of the laboratory room when experiment took place was high, 24-27C). In this work we proposed the use of very small thermal camera with small spatial resolution that is used for smart glasses. We also compared two different estimators: one operating in frequency domain, the second one in time domain. Results proved high accuracy of the method and both estimators. However, in this study we used manually selected ROI for nostril area. This can be performed automatically using automatic face detection for thermal images and detection of nostril regions [20]. This will be a subject for further study. It is also important to investigate different signal quality measures that can be used to evaluate if the measured signal (or RR value) is more or less reliable. Such possible measures can analyze the spectral purity of the periodical components (e.g. Hijorth parameters [21], etc.) or other similar parameters (goodness of fit for parametric models [22], periodicity of the autocorrelation function, etc.).

Using smart glasses senses of a healthcare professional could be extended providing additional knowledge about a patient acquired during routine interviews. This is more natural method of observation because it does not influence the patient by using (wearing) additional equipment. Additionally, smart glasses can provide very good source of medical data for self-diagnostics of the smart glasses user. However, practical application of such methods requires comfortable design of smart glasses, which is a task for further research.

\section{REFERENCES}

[1] Smart Glasses Portal, 2016, Available: http://www. smartglassesnews.org.

[2] Evena Medical, 2014, Available: http://evenamed.com/ even5672/ products/glasses.

[3] C. Borchers, "Google Glass moves into the hospital at Beth Israel", the Boston Globe, 14.06.2014, Available: http://www.bostonglobe.com

[6] J. Ruminski, A. Bujnowski, T. Kocejko, A. Andrushevich, M. Biallas, R. Kistler, "The data exchange between smart glasses and healthcare information systems using the HL7 FHIR standard", 9th International Conference on Human System Interaction, IEEE, eXplore, 2016.

[6] J. Ruminski, K. Czuszynski, "Application of smart glasses for fast and automatic color correction in health care", Proc. of the 37th Annual International Conference of the IEEE Engineering in Medicine and Biology Society, Milan, Italy, 2015.

[6] Philips, "Delivering vital patient data via Google Glass", 03.10.2013, Available: http://www.healthcare.philips.com/main/about/future-ofhealthcare/

[7] J. Hernandez, Y. Li, J. M. Rehg and R. W. Picard, "BioGlass: Physiological parameter estimation using a head-mounted wearable device," Wireless Mobile Communication and Healthcare (Mobihealth), 2014 EAI 4th International Conference on, Athens, 2014, pp. 55-58.

[8] J. Ruminski, "The accuracy of pulse rate estimation from the sequence of face images", 9th International Conference on Human System Interaction, IEEE, eXplore, 2016.

[9] M. Z. Poh, D. J. McDuff, R. W. Picard "Non-contact, automated cardiac pulse measurements using video imaging and blind source separation", Opt. Expr., vol. 18, pp.10762 -10774, 2010.

[10] J. Ruminski, M. Smiatacz, A. Bujnowski, A. Andrushevich, M. Biallas, R. Kistler, "Interactions with recognized patients using smart glasses," in Human System Interactions (HSI), 2015 8th International Conference on, pp.187-194, 25-27 June 2015

[11] K. Czuszynski, J. Ruminski, "Interaction with medical data using QRcodes", in Human System Interactions (HSI), 2014 7th International Conference on, pp.182-187, 16-18 June 2014.

[12] A. Kwasniewska, J. Klimiuk-Myszk, J. Ruminski, J. Forrier, B. Martin, I. Pecci, "Quality of graphical markers for the needs of eyewear devices," in Human System Interactions (HSI), 2015 8th International Conference on , vol., no., pp.388-395, 25-27 June 2015.

[13] P. Przystup, A. Bujnowski, J. Ruminski, J. Wtorek, "A multisensor detector of a sleep apnea for using at home", The 6th HSI Conference, IEEE Xplore, pp. 513-517, 2013.

[14] F. Zhao, M. Li, Y. Qian, J. Z. Tsien, "Remote Measurements of Heart and Respiration Rates for Telemedicine", PLoS One. 2013; 8(10).

[15] M. Z. Poh, D. J. McDuff, P. W. Picard, "Advancements in noncontact, multiparameter physiological measurements using a webcam". IEEE Trans Biomed Eng 58: 7-11, 2011.

[16] A. K. Abbas, K. Heimann, K. Jergus, T. Orlikowsky, S. Leonhardt, "Neonatal non-contact respiratory monitoring based on real-time infrared thermography”, BioMedical Engineering OnLine, vol. 10:93, BioMed Central, 2011.

[17] E. A. Bernal, L.K. Mestha, E. Shilla, "Non contact monitoring of respiratory function via depth sensing," in Biomedical and Health Informatics (BHI), 2014 IEEE-EMBS International Conference on, pp.101-104, 1-4 June 2014.

[18] R. Murthy, I. Pavlidis , "Non-contact monitoring of respiratory function using infrared imaging," IEEE Engineering in Medicine and Biology Magazine. vol.25, pp.57-57, 2006.

[19] J. Ruminski, "Evaluation of the respiration rate and pattern using a portable thermal camera", Proc. of the 13th Quantitative Infrared Thermography Conference, Gdansk 2016.

[20] A. Kwasniewska, J. Ruminski, „Real-time facial feature tracking in poor quality thermal imagery", Proc. of the 9th International Conference on Human System Interaction, IEEE, eXplore, 2016.

[21] L. Sörnmo, P. Laguna, "Bioelectrical Signal Processing in Cardiac and Neurological Applications”, Academic Press, 2005.

[22] J. Ruminski, B. Bobek-Billewicz, "Parametric imaging in dynamic susceptibility contrast MRI-phantom and in vivo studies," IEMBS'04. 26th Annual International Conference of the IEEE, pp. 1104-1107, 2004. 